\title{
Android Internet Traffic Classification Based on Bayesian Analysis Techniques
}

\author{
luo dawei ${ }^{1, a}$,han $z_{i j i a n^{1, a}}$,chen shangshu ${ }^{1, a}$,liu shangdong ${ }^{1, a}$ \\ ${ }^{1}$ Nangjing University of Posts and Telecommunications Jiangsu Nanjing 210046,china \\ aeiluodawei@sina.com
}

KEYWORD: traffic classification, Naive Bayesian, Android data flow

\begin{abstract}
The wireless telecommunication techniques based on android operation system has been widely used both in life and work. It is of huge importance to classify and monitor the accurate android network traffic . However, the appliance of port jump technique and port encryption technique make classifying network traffic based on port type become much less effective. Here we put forward a method based on Naive Bayesian Analysis to classify different Android application, which combines the features of network data flow and Naive Bayesian. The experimental results show that this method has high classification accuracy.
\end{abstract}

\section{Outline}

According to the latest third quarter report from Strategy Analytics, Android operating system ranked first in the mobile operating system market with $83.6 \%$ market share. So we can see that the mobile network applications based on the platform of Android operating system have already become the mainstream in the market and the applications in Android operating system will increase in large numbers in the future. Thus study and analysis of Android Internet traffic classification has great significance.

However, due to the growing complication of mobile applications and Internet traffic, some emerging mobile terminal business are using technologies such as dynamic port and port encryption. These make the traditional port-based and playload-based Internet traffic classification methods inefficient. This paper presents a new method that is based on Naive Bayesian method (NB) to classify and analyze Android Internet traffic. The classification works in accordance with property of the Internet traffic data.

\section{Traffic classification based on Naive Bayesian}

\subsection{Naive Bayesian}

Naive Bayesian is a kind of distribution function that combines the categories and properties. It estimates the category of experiment samples by calculating the joint probability of them.

Naive Bayesian under normal circumstance: Assume that the sample space of random experiment $E$ is $S, B_{1}, B_{2}, \ldots, B_{n}$ is a divide of sample space $S, A$ is an event and $P(A)>0$,here is the formula:

$$
P\left(B_{i} \mid A\right)=\frac{P\left(B_{i}\right) P\left(A \mid B_{i}\right)}{\sum_{j=1}^{n} P\left(B_{j}\right) P\left(A \mid B_{j}\right)} i=1,2, \ldots, n
$$

$P\left(B_{i}\right)$ is called prior probability. We can achieve that by experience. The $P\left(B_{i} \mid A\right)$ got from the formula above is called posterior probability ${ }^{[2]}$.

\subsection{Naive Bayesian applied in data flow}

When using Naive Bayesian to identify and classify internet traffic, we assume the classification of traffic flow $F$ is $\left\{C_{1}, C_{2}, \ldots, C_{k}\right\}$, and $k$ represents the number of categories of traffic. If randomly given a internet flow $F$, the formula of conditional probability of that flow $F$ belongs to category $C_{k}$ is: 


$$
P\left(C_{k} \mid F\right)=\frac{P\left(F \mid C_{k}\right) P\left(C_{k}\right)}{P(F)}
$$

$P\left(C_{k}\right)$ is the prior probability of category $C_{k}$, it can be acquired by the proportion of flow $C_{k}$ in the whole data flow. $P(F)$ is a normal constant, which signifies the marginal probability of flow $F$. $P\left(F \mid C_{k}\right)$ is the conditional probability of that flow $F$ belongs to category $C_{k}$, we can get it from training data. Furthermore, the properties of traffic flow $F$ can be simplified into the property eigenvector $\left(F_{1}, F_{2}, \ldots, F_{n}\right)^{T}$. So the probability $P\left(F \mid C_{k}\right)$ can be explained by formula (2-3)

$$
P\left(F \mid C_{k}\right)=P\left(F_{1}, F_{2}, \ldots, F_{n} \mid C_{k}\right)
$$

According to the assumption of prior condition of Naive Bayesian, the properties of internet flow are independent to each other and they follow Gaussian distribution ${ }^{[3]}$. As a result of that, formula(2-3)can be simplified into(2-4)

$$
P\left(F_{1}, F_{2}, \ldots, F_{n} \mid C_{k}\right)=\prod_{j=1}^{n} P\left(F_{j} \mid C_{k}\right)
$$

Then we apply (2-4) into (2-3) we can get (2-5)

$$
P\left(C_{k} \mid F_{1}, F_{2}, \ldots, F_{n}\right)=\frac{\prod_{j=1}^{n} P\left(F_{j} \mid C_{k}\right) P\left(C_{k}\right)}{\sum_{k=1}^{m} \prod_{j=1}^{n} P\left(F_{j} \mid C_{k}\right) P\left(C_{k}\right)}
$$

\section{Collecting sample of data flow}

\subsection{Choosing properties of data flow}

Because the number of data we collected is so huge, we need to select appropriate and typical data flow so that we can apply them into Naive Bayesian, in which way can we avoid the cumbersome calculating process and ensure the accuracy of the Naive Bayesian as well. We choose five properties ${ }^{[4]}$ as shown in Table 1.

Table 1 properties selected of data flow

\begin{tabular}{|l|l|}
\hline Property 1 & Duration of flow \\
\hline Property 2 & Total number of packets \\
\hline Property 3 & Total number of flows \\
\hline Property 4 & Average number of bytes in each packet \\
\hline Property 5 & Time intervals between each packet \\
\hline
\end{tabular}

\subsection{Collecting data}

We use wireshark network packet analysis software ${ }^{[5]}$ to grab the network data packets on computers in the lab. Then we connect several mobile equipments with Android operating system to the wireless network generated by the computers and at the same time, run different network applications such as wechat, weibo and so on. W e can finally get network packet in the form of pcap $^{[6]}$

\subsection{Experiment tools and data handle}

After collecting enough data packets, we use JNetPcap ${ }^{[8]}$ to analyze the data flow based on Naive Bayesian. At first we install the API library in eclipse and set up JNetPcap. When we finish setting up the professional environment, we are able to write Java code to read the packets 
whose form is pcap ${ }^{[9]}$ coming from wireshark .Finally, we are able to print the content of data packets and collect the statistics of data properties.

The data we analyze in the essay are all real. We continuously collected data for six hours from 10am to $4 \mathrm{pm}$ on July 22th, 2015. 10928.54 $M$ data was got in total as the sample data. It contains 68940 network flows generating by sex different types of application. All the data we got is shown in Table 2.

Table 2 data collected in lab

\begin{tabular}{|l|r|r|r|}
\hline application & $\begin{array}{l}\text { Number } \\
\text { of flows }\end{array}$ & $\begin{array}{l}\text { Number } \\
\text { of } \\
\text { packets }\end{array}$ & Average bytes \\
\hline youku video & 2844 & 70524 & 129821.6 \\
\hline wechat & 5688 & 83088 & 733.6 \\
\hline weibo & 35928 & 583416 & 319.7 \\
\hline QQ & 10440 & 1112184 & 776.4 \\
\hline UC browser & 10440 & 367704 & 517.3 \\
\hline baidu music & 3600 & 992448 & 783.7 \\
\hline
\end{tabular}

\section{Result analysis}

The indicators used to estimate the efficiency of method are accuracy and overall accuracy . The accuracy is used to identify single application and overall accuracy is used to identify the whole sample.

Assume that the number of classifying correctly is $N_{r}$ and that of classifying wrong is $N_{w}$,we have:

$$
\begin{gathered}
\text { accuracy }=\frac{N_{r}}{N_{r}+N_{w}} \\
\text { overall accuracy }=\frac{\sum N_{r}}{\sum N_{r}+\sum N_{w}}
\end{gathered}
$$

\subsection{Classification of the whole sample}

When we analyze the experiment data, it is of importance to choose appropriate training data and testing data. It will help us to know about the stability and tendency of this method. We decided to choose separately $80 \%, 50 \%$ and $20 \%$ as the training data, the corresponding data left as the testing one. The results are shown in Table 3

Table 3 overall accuracy for $N B$

\begin{tabular}{|l|r|r|r|}
\hline $\begin{array}{l}\text { Training } \\
\text { data }\end{array}$ & minimum & maximum & Average \\
\hline $20 \%$ & $38.58 \%$ & $81.13 \%$ & $59.86 \%$ \\
\hline $50 \%$ & $53.14 \%$ & $87.67 \%$ & $70.41 \%$ \\
\hline $80 \%$ & $62.17 \%$ & $88.95 \%$ & $75.56 \%$ \\
\hline
\end{tabular}

We can clearly see that In the table the prior probability changes when the number of training data grow from $20 \%$ to $80 \%$. In this process the overall accuracy for $N B$ rises. However, the rising is relatively drastic, which means that $N B$ is lack of stability and the accuracy itself is relatively low. 


\section{2 classification of single application}

In order to study the accuracy of data analysis of $N B$, we also need to work on the accuracy for single application. We set up the mathematical model using $80 \%$ of sample data as the training data. The result is shown in table 4

Table 4 the accuracy for classifying different application

\begin{tabular}{|l|l|l|l|l|l|l|}
\hline application & $\begin{array}{l}\text { youku } \\
\text { video }\end{array}$ & wechat & weibo & QQ & $\begin{array}{l}\text { UC } \\
\text { browser }\end{array}$ & $\begin{array}{l}\text { baidu } \\
\text { music }\end{array}$ \\
\hline accuracy & $80.70 \%$ & $47.90 \%$ & $77.00 \%$ & $54.60 \%$ & $92.10 \%$ & $86.20 \%$ \\
\hline
\end{tabular}

The conclusion we can get from table 4 is that the difference $N B$ make about the accuracy of different application is big, especially when working on the complicated application such as wechat who has many functions.

\section{Conclusion}

As Android operating system becoming the mainstream in the market, developing Android Internet traffic monitoring and analysis technology is of great significance in Internet management and supervision. This paper presents a new Android Internet traffic classification method based on Naive Bayesian method. According to the data we can see that NB method can be used in Android Internet traffic classification. However, this method has its disadvantages, e.g. Lack of stability, relatively low accuracy etc. One of its reason is that in NB method, the properties of Internet traffic only obey Gauss distribution while there are more complex relationships among them. Thus we can improve the NB method in this direction to make the classification more accurate in the future.

\section{Reference}

[1] Moore A W, Papagiannaki K Toward the accurate identification of network applications[C]//Proceeding of Sixth Passive and Active Measurement Workshop(PAM 2005). Springer-VerlagLNCS,2005:41-45

[2] Kong Gaohua, He Ming etc. Probability and Stochastic Processes.People's Posts and Telecommunications Press, 2012:18-19

[3] Chang Jianping, Li Hailin etc. Random signal analysis. Science Press, 2006:30-32

[4] A.W.Moore and D.Zuev. Discriminators for use in flow-based classification Technical report, Intel Research,Cambridge,2005

[5] Wang Xiaohui etc. Practical Packet Analysis: Using Wireshark to Solve Real-World Network Problems. Tsinghua University Press, 2015:122-134

[6] Xu Yingkang, Zhang Ali. Design of network data packet analysis software based on PCAP.Modern Electronic Technology, 2013 (10) : 49-51

[7] Li Han, Liang Wei. Research on network traffic identification method.Communications Technology, 2008,41 (11) : 88-90

[8] Mark Bednarczyk. JNetPacp[EB/OL]. http://www.jnetpcap.com/

[9] Mark Bednarczyk. Packet flows[EB/OL].http://jnetpcap.com/node/144

2015-07-21

[10] A.McCallum and K.Nigam. A comparison of event models for naive bayes text classification. In In AAAI-98 Workshop on Learning for Text Categorization,1998 